\title{
A reliable transportation problem
}

\section{Abdul Quddoos}

Department of Mathematics, Integral University, Lucknow, India

\begin{abstract}
In today's market, managers are being pressurized to deliver the product in a reliable and cost effective manner. The aim of the present work is to model a transportation problem where decision maker has a flexibility to set his own reliability level when he minimizes the overall transportation cost. To achieve this goal, the overall reliability of transportation system is calculated and a mathematical model of transportation problem is formulated. A numerical experiment is also conducted to illustrate the proposed model.
\end{abstract}

Keywords: Transportation Problem; Reliability

\section{Introduction}

The reliability of a network system can be defined as the ability of the system to perform a required set of actions under the stated conditions without any failure. The study of network reliability started in the field of communication network and then penetrated into many areas such as electric network, computer network and railway network and facility location problems etc. Hamedi et al. (2012) focussed on routing and scheduling of a fleet of trucks for humanitarian distribution over an unreliable road network. Aggarwal et al. (1982) gave an algorithm for obtaining an optimal network topology of a large scale computer communication network which gives maximum s-t reliability with the specified cost of establishment of links. Chopra et al. (1984) did the same with an additional advantage that the system need not to be redesigned when the cost of establishment of links are enhanced. Zheng et al. (2011) studied the carrying capacity reliability of railway networks in China. Drezner (1986) was the first researcher who considered facility location problem with unreliable supplier. Synder and Dakins (2005) presented a model for choosing facility location. Their goal was to choose facility locations which are more reliable and less expensive. Many other researchers have been presented studies on facility location problem with the assumption that facilities are subjected to reliability. Some of them are Tomlin (2006), Synder et al. (2010), Qi and Shen (2007), Qi et al. (2010). But to the best of our knowledge no paper is available in literature regarding the reliability of classical transportation problem (TP) proposed by Hitchcock (1941) which shows a research gap in the studies of classical TP. In present scenario, the markets are being highly competitive which compel the managers of service systems to deliver the services in a cost effective manner with maximum reliability level. The managers dealing with supply chain related issues always try to find out the ways to deliver the products in a reliable and cost effective manner in order to stay competitive.

In this paper we consider a classical transportation problem proposed by Hitchcock (1941) and refer this transportation system as Hitchcock Transportation System (HTS). In many practical situations, it becomes very challenging for managers to transport the products to a given destination within a specified period of time due to land slide, heavy traffic, bad condition of roads etc. So we computed the overall reliability of HTS by considering the route reliability in terms of travel time reliability (the travel time reliability is concerned with the probability that a trip between a given Origin-Destination pair will be made successfully within a given interval of time) and formulated a Reliable Hitchcock Transportation Problem. We give a numerical example in order to validate the proposed formulation.

\section{Transportation Problem}

In a classical Hitchcock (1941) transportation problem, a homogeneous product is to be transported from ' $\mathrm{m}$ ' sources to 'n' destinations. The amount of product available at source $S_{i}, i=1,2, \cdots, m$ is $a_{i,} i=1,2, \cdots, m$ and the quantity 
demanded at destination $D_{i}, j=1,2, \cdots, n$ is $b_{i .} j=1,2, \cdots n . C_{i i}$ is the cost of transporting a unit of product from source $S_{i}, i=1,2, \cdots, m$ to destination $D_{i}, j=1,2, \cdots, n$.Thus the Hitchcock transportation problem is mathematically expressed as:

\section{Problem 1:}

Minimize: $Z=\sum_{i=1}^{m} \sum_{j=1}^{n} C_{i j} x_{i j}$

subject to;

$\sum_{j=1}^{n} x_{i j}=a_{i}, i=1,2 \ldots m($ Row Restriction $)$

$\sum_{i=1}^{m} x_{i j}=b_{j}, j=1,2 \ldots n($ Column Restriction $)$

$x_{i j} \geq 0, i=1,2 \ldots m, j=1,2 \ldots n$.

The physical structure of Problem 1 may be considered as a Transportation System (TS) which we call HTS. The HTS is depicted in the following figure 1.

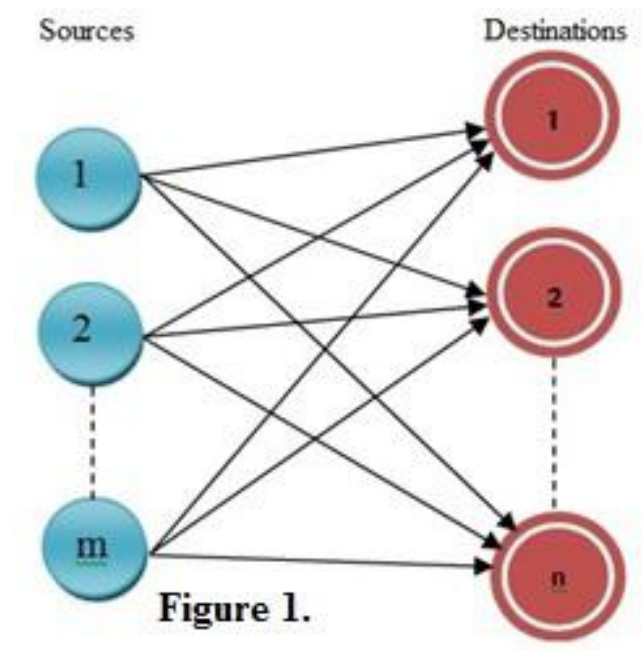

\subsection{Necessary condition of HTS:}

Necessary condition: A positive amount of product must be transported from each source $S_{i}, i=1,2, \cdots, m$ to at least one destination $D_{i}, j=1,2, \cdots, n$; such that the total supply of each source must be exhausted and the total demand at each destination must be satisfied.

\section{Computation of overall reliability of HTS}

Let us consider that $r_{i j}, i=1,2, \cdots, m ; j=1,2, \cdots, n$ be the travel time reliability of $\operatorname{link}(i, j)$, where $\operatorname{link}(i, j)$ represents the route from source $S_{i}, i=1,2, \cdots, m$ to destination $D_{i}, j=1,2, \cdots, n$ It can be seen from figure 1, that, from source $S_{1}$ to every destination $D_{j} j=1,2, \cdots, n$ there exist ' $n$ ' number of links [i.e., $\operatorname{link}(1,1), \operatorname{link}(1,2), \cdots, \operatorname{link}(1, n)]$. Similarly, from source $S_{2}$ to every destination $D_{i}, j=1,2, \cdots, n$ again there exist ' $n$ ' number of links [i.e., $\operatorname{link}(2,1), \operatorname{link}(2,2), \cdots, \operatorname{link}(2, n)]$ and so on. So the total number of links from each source $S_{i}, i=1,2, \cdots, m$ to every destination be ' $m n$ '.

In view of necessary condition given in section (2.1) is can be seen that among ' $N$ ' links, atleast one link must be selected to ship the product from first source $S_{1}$ to any destination i.e. atleast one link must be in operating state. So it may be considered as a subsystem (say, Subsystem 1) [see figure 2.] having ' $n$ ' components operating in parallel. i.e., the subsystem will work if atleast one of its components is working. 

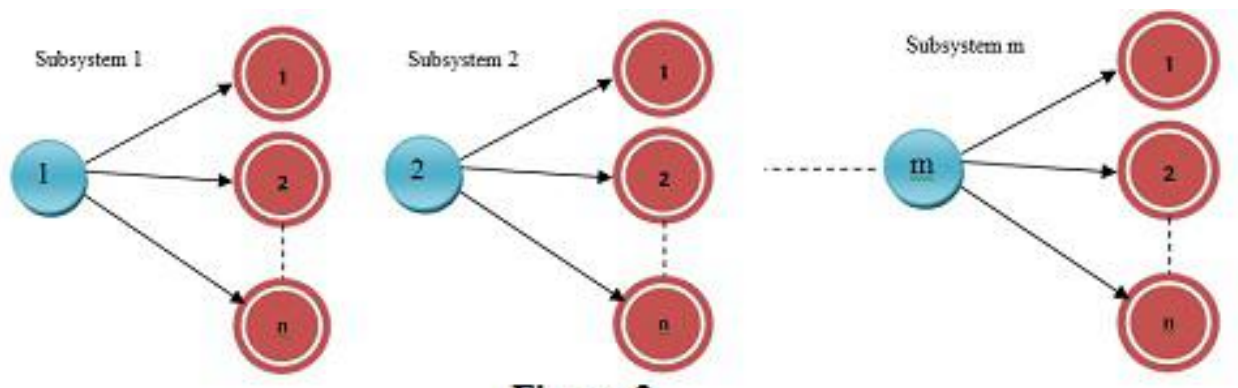

Figure 2.

The reliability $\mathrm{R}_{1}$ of Subsystem 1 may be given as follows:

$$
R_{1}=1-\prod_{j=i}^{n}\left(1-r_{1 j}\right)
$$

Where, $\operatorname{link}(1, j), j=1,2, \ldots, n$ may be treated as components of Subsystem-1 with reliability $r_{i}, j=1,2, \ldots, n$ Similar subsystems can also be considered for remaining (m-1) sources [see figure 2.] and reliability of subsequent subsystems can be represented as follows:

$$
R_{i}=1-\prod_{j=i}^{n}\left(1-r_{i j}\right), \quad i=2,3, \cdots, m
$$

The general expression for reliability of a Subsystem $\mathrm{i}, \mathrm{i}=1,2, \ldots, \mathrm{m}$ can be obtained by combining equation (1) and (2) together as,

$$
R_{i}=1-\prod_{j=i}^{n}\left(1-r_{i j}\right), \quad i=1,2, \cdots, m
$$

It can further be seen from necessary condition that HTS works if and only if all subsystems are working. So, it may be treated as a system having ' $m$ ' subsystems operating in series. Thus the overall reliability $R$ of HTS is,

$$
\begin{aligned}
& R=\prod_{j=i}^{n} R_{i}, \quad i=1,2, \cdots, m \\
& \text { or } \\
& R=\prod_{i=1}^{m}\left(1-\prod_{j=i}^{n}\left(1-r_{i j}\right)\right)
\end{aligned}
$$

\section{Formulation of Reliable Hitchcock Transportation Problem (RHTP)}

It is well known fact that Hitchcock Transportation Problem is concerned with minimizing the total cost of transportation. But in real life situations, one may be interested in minimizing the total cost of transportation subject to a desired (pre-specified) level of overall reliability of HTS. A Reliable Hitchcock Transportation Problem is formulated as follows,

Let us define the decision variables,

$x_{i j}=$ Amount of product shipped from source $S_{i}$ to destination $D_{j}$

$y_{i j}= \begin{cases}1 & \text { If destination } D_{j} \text { receives supply from source } S_{i} \text { [or } \operatorname{link}(i, j) \text { is selected] } \\ 0 & \text { Otherwise }\end{cases}$

In order to compute the overall reliability of HTS, we associate a binary variable $y_{i i}$ with every $r_{i j}$ which ensures 
that the reliability of only those links are taken into account for which $x_{i j}>0$. Then equation (4) becomes,

$$
R=\prod_{i=1}^{m}\left(1-\prod_{j=i}^{n}\left(1-r_{i j} \cdot y_{i j}\right)\right)
$$

Using equation (5), the Reliable Hitchcock Transportation Problem may be formulated as:

\section{Problem 2:}

Minimize : $Z=\sum_{i=1}^{m} \sum_{j=1}^{n} C_{i j} x_{i j}$

Subject to;

$\sum_{j=1}^{n} x_{i j}=a_{i}$

$\sum_{i=1}^{m} x_{i j}=b_{j}$

$R=\prod_{i=1}^{m}\left(1-\prod_{j=1}^{n}\left(1-r_{i j} \cdot y_{i j}\right)\right) \geq R_{\alpha}$

$y_{i j} \leq x_{i j} \leq \min \left(a_{i}, b_{j}\right) \cdot y_{i j}$

$y_{i j} \in\{0,1\}$ and integer $x_{i j} \geq 0 ; i=1,2, \cdots, m ; j=1,2, \cdots, n$.

The objective function minimizes the total cost of sending supply from source $S_{i}$ to destination $D_{i}$. Constraint (ii) and (iii) are same as in classical Hitchcock Transportation Problem. Constraint (iv) ensures that the overall HTS reliability is not less than a pre-specified reliability level $R_{\alpha} \leq R_{0}$, where $R_{0}$ is the maximum reliability HTS. $R_{0}$ may be obtained by putting $y_{i j}=1, \forall i$, and $j$ in equation (5). Constraint (v) ensures, $y_{i j}=1$, when $x_{i j}>0$ and $y_{i j}=0$, when $x_{i j}=0$. The constraint (vi) provides the non-negativity restriction on amount to be supplied (i.e. $x_{i j}$ ) and binary integer restriction of indicator function $y_{i i}$.

\section{Numerical Example}

Following numerical example is considered to illustrate the Reliable Hitchcock Transportation Problem:

\begin{tabular}{|c|c|c|c|c|c|}
\hline $\begin{array}{l}\text { Destinations- } \\
\text { Sources } \downarrow\end{array}$ & $\mathrm{D}_{1}$ & $\mathrm{D}_{2}$ & $\mathrm{D}_{3}$ & $\mathrm{D}_{4}$ & Availability $\left(a_{i}\right)$ \\
\hline $\mathrm{S}_{1}$ & {$[03,0.7]$} & {$[05,0.8]$} & {$[07,0.85]$} & {$[2,0.65]$} & 10 \\
\hline $\mathrm{S}_{2}$ & {$[08,0.86]$} & {$[06,0.83]$} & {$[05,08]$} & {$[03,0.7]$} & 08 \\
\hline $\mathrm{S}_{3}$ & {$[10,0.95]$} & {$[09,0.93]$} & {$[02,0.65]$} & {$[05,0.8]$} & 12 \\
\hline Demand $\left(b_{j}\right)$ & 09 & 10 & $\mathbf{0 7}$ & 04 & 30 (Total) \\
\hline
\end{tabular}

Table 1

Substituting, $y_{i j}=1, i=1,2,3$, and $j=1,2, \ldots 4$, and the corresponding links reliabilities $\left(r_{i j}\right)$ in equation (7) we get $R_{0}=0.9951826$. So the maximum $99.51826 \%$ reliability of the above HTS can be achieved.

The above problem can be mathematically expressed as:

\section{Problem 3:}

Minimize : $Z=3 x_{11}+5 x_{12}+7 x_{13}+2 x_{14}+8 x_{21}+6 x_{22}+5 x_{23}+3 x_{24}+10 x_{31}+9 x_{32}+2 x_{33}+5 x_{34}$ Subject to; 


$$
\begin{aligned}
& x_{11}+x_{12}+x_{13}+x_{14}=10 \\
& x_{21}+x_{22}+x_{23}+x_{24}=8 \\
& x_{31}+x_{32}+x_{33}+x_{34}=12 \\
& x_{11}+x_{21}+x_{31}=9 \\
& x_{12}+x_{22}+x_{32}=10 \\
& x_{13}+x_{23}+x_{33}=7 \\
& x_{14}+x_{24}+x_{34}=4 \\
& R=\left[1-\left\{\left(1-0.70 y_{11}\right)\left(1-0.80 y_{12}\right)\left(1-0.85 y_{13}\right)\left(1-0.65 y_{14}\right)\right\}\right] \\
& \quad \quad \times\left[1-\left\{\left(1-0.86 y_{21}\right)\left(1-0.83 y_{22}\right)\left(1-0.8 y_{23}\right)\left(1-0.7 y_{24}\right)\right\}\right] \\
& \quad \quad \times\left[1-\left\{\left(1-0.95 y_{31}\right)\left(1-0.93 y_{32}\right)\left(1-0.65 y_{33}\right)\left(1-0.8 y_{34}\right)\right\}\right] \geq R \\
& y_{11} \leq x_{11} \leq 9 y_{11} \\
& y_{12} \leq x_{12} \leq 10 y_{12} \\
& y_{13} \leq x_{13} \leq 7 y_{13} \\
& y_{14} \leq x_{14} \leq 4 y_{14} \\
& y_{21} \leq x_{21} \leq 8 y_{21} \\
& y_{22} \leq x_{22} \leq 8 y_{22} \\
& y_{23} \leq x_{23} \leq 7 y_{23} \\
& y_{24} \leq x_{24} \leq 4 y_{24} \\
& y_{31} \leq x_{31} \leq 9 y_{31} \\
& y_{32} \leq x_{32} \leq 10 y_{32} \\
& y_{33} \leq x_{33} \leq 7 y_{33} \\
& y_{34} \leq x_{34} \leq 4 y_{34} \\
& y_{i j} \in\{0,1\} \text { and integer } x_{i j} \geq 0 ; i=1,2,3 j=1,2, \cdots, 4 \\
& \\
& \quad P 15
\end{aligned}
$$

Problem 3 is solved by using Lingo 13.0 software package for different pre-specified levels of overall reliability of HTS and the results so obtained are recorded in table 2 as follows,

Table 2

\begin{tabular}{c|l|l}
\hline S. No. & Minimized Cost (Objective Function) & Pre-specified reliability $\left(R_{\alpha} \leq R_{0}=0.9951826\right)$ \\
\hline 1. & 123 & 0.75 \\
2. & 124 & 0.80 \\
3. & 124 & 0.85 \\
4. & 127 & 0.90 \\
5. & 128 & 0.95 \\
6. & 147 & 0.99 \\
7. & Infeasible & 0.996 \\
\hline
\end{tabular}




\section{Discussion and Conclusion}

As shown in table 2, different minimum values of total cost of transportation are obtained for different levels of HTS reliabilities. It can also be observed that the problem become infeasible when $R a$ exceeds $R 0$. Thus the HTP is redefined with an additional reliability constraint. This additional constraint enables the decision maker to set his own reliability level with the maximum limit $R_{0}$. With the help of proposed Reliable Hitchcock Transportation Problem decision maker becomes capable for obtaining the optimal shipment policy with a desired overall reliability level of transportation system. So the proposed Reliable Hitchcock Transportation Problem may be proven very helpful for decision makers to design the optimal shipment policy with a pre-specified reliability level.

\section{References}

1. Aggarwal KK, Chopra YC, Bajwa JS. Topological layout of links for optimizing the s-t reliability in a computer communication network. microelectronics reliability 1982; 22(03): 341-345.

2. Chopra YC, Sohi BS, Tiwari RK. Network topology for maximizing the terminal reliability in a computer communication network. Microelectronics Reliability 1984; 24(05): 911-913.

3. Drezner Z. Heuristic solution methods for two location problems with unreliable facilities. Journal of the Operational Research Society 1987; 38(6): 509-514.

4. Hitchcock FL. The Distribution of a product from several sources to numerous localities. Journal of Mathematics and Physics 1941; 20: 224-230.

5. Hamedi M, Haghani A, Yang S. Reliable transportation of humanitarian supplies in disaster response: model and heuristic. Procedia - Social and Behavioral Sciences 2012; 541205-1219.

6. Qi L, Shen ZJM, Snyder LV. The effect of supply disruptions on supply chain design decisions. Transportation Science 2010; 44(2): 274-289.

7. Qi L, Shen ZJM. A supply chain design model with unreliable supply. Naval Research Logistics 2007; 54(8) 829-844.

8. Snyder LV, Daskin MS. Reliability models for facility location: The expected failure cost case. Transportation Science 2005; 39(3): 400-416.

9. Tomlin BT. On the value of mitigation and contingency strategies for managing supply chain disruption risks. Management Science 2006; 52(5): 639-657.

10. Zheng Y, Zhang X, Xu B. Carrying capacity reliability of railway networks. Journal of Transportation Systems Engineering and Information Technology 2011; 11(4): 16-21. 